\title{
Bilateral adrenal hemorrhages in a patient with heparin-induced thrombocytopenia
}

\author{
Mario Montealegre-Gallegos MD, Somnath Bose MD
}

— Cite as: CMAJ 2019 November 4;191:E1222. doi: 10.1503/cmaj.190379

A 72-year-old man with a history of type 2 diabetes mellitus and atrial fibrillation was admitted to our institution with a non-ST-elevation myocardial infarction. Intravenous heparin therapy was started. Cardiac catheterization showed multivessel coronary artery disease, and he underwent urgent triple coronary artery bypass surgery with left atrial appendage ligation. He was discharged on postoperative day 4 but was readmitted that same day with atrial fibrillation with rapid ventricular rate and respiratory failure.

On postoperative day 7 ( $9 \mathrm{~d}$ after administration of the initial heparin dose), the patient was found to be thrombocytopenic (platelet nadir $38000 / \mathrm{mm}^{3}$ ), with PF4-heparin antibodies (2.41 optical density). After confirmation with a serotonin-release assay, heparin-induced thrombocytopenia was diagnosed. Heparin was discontinued, and anticoagulation was maintained with bivalirudin.

Two days after bivalirudin was started, the patient became profoundly hypotensive, requiring an escalating dosage of vasopressors. After excluding common causes of shock after cardiac surgery (e.g., hemothorax, tamponade), we performed abdominal computed tomography without contrast to evaluate for other potential causes of persistent hypotension; this study showed bilateral adrenal hemorrhages (Figure 1; Appendix 1, available at www.cmaj.ca/ lookup/suppl/doi:10.1503/cmaj.190379/-/DC1). Primary adrenal insufficiency secondary to adrenal hemorrhage was thought to be the most likely cause of shock. A stimulation test with cosyntropin, $250 \mu$ given intravenously, was diagnostic of adrenal insufficiency (baseline cortisol level $30 \mathrm{nmol} / \mathrm{L}$, level after cosyntropin administration $28 \mathrm{nmol} / \mathrm{L}$ ). Treatment was started with hydrocortisone, $100 \mathrm{mg}$ administered intravenously every 8 hours, which led to rapid improvement in the patient's hemodynamic status, and he was subsequently weaned off vasopressor support.

Heparin-induced thrombocytopenia is a well-established complication of heparin treatment that may result in paradoxical arterial and venous thromboses. ${ }^{1}$ Adrenal hemorrhage is an infrequent complication of heparin-induced thrombocytopenia and is likely secondary to occlusive venous thrombosis. ${ }^{2}$ In our patient, bilateral adrenal hemorrhages presented as acute adrenal insufficiency causing shock. Clinicians should be aware of the possibility of adrenal hemorrhages presenting as undifferentiated shock in the setting of heparin-induced thrombocytopenia.

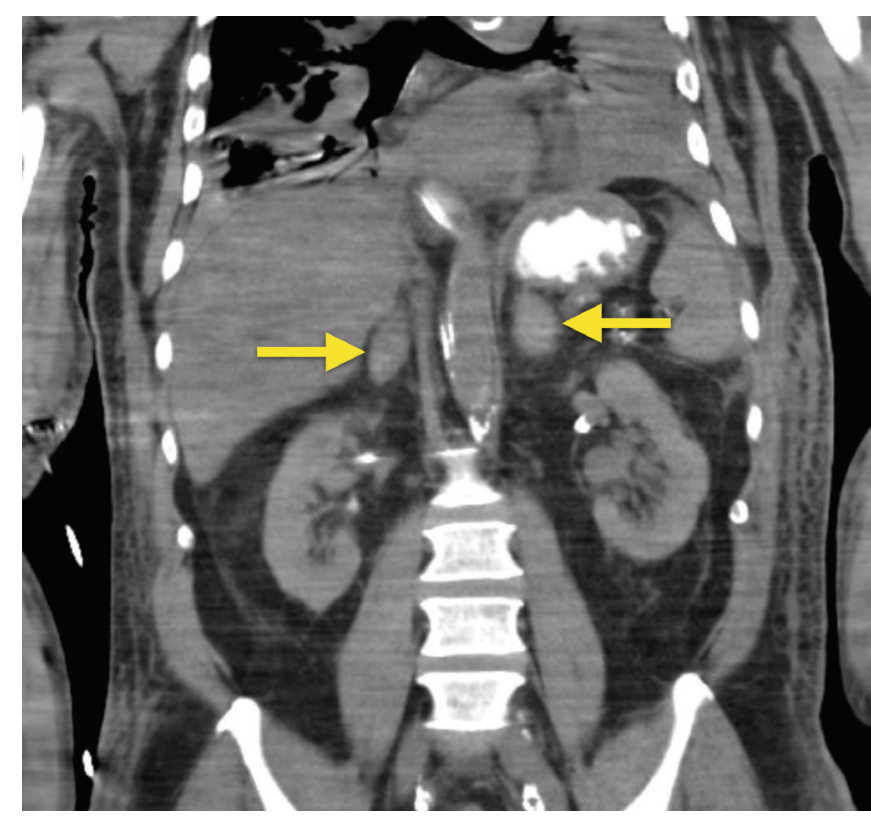

Figure 1: Coronal noncontrast computed tomography scan of a 72-yearold man with shock, showing bilateral adrenal hematomas (arrows) with densities ranging from 71 to 73 Hounsfield units. The left hematoma measured $39 \times 25 \mathrm{~mm}$, and the right hematoma, $36 \times 25 \mathrm{~mm}$.

\section{References}

1. Rosenberger LH, Smith PW, Sawyer RG, et al. Bilateral adrenal hemorrhage: the unrecognized cause of hemodynamic collapse associated with heparininduced thrombocytopenia. Crit Care Med 2011;39:833-8.

2. Warkentin TE, Safyan EL, Linkins LA. Heparin-induced thrombocytopenia presenting as bilateral adrenal hemorrhages. N Engl J Med 2015;372:492-4.

\section{Competing interests: None declared.}

This article has been peer reviewed.

The authors have obtained patient consent.

Affiliation: Department of Anesthesia, Critical Care and Pain Medicine, Beth Israel Deaconess Medical Center, Boston, Mass.

Correspondence to: Somnath Bose, sbose2@bidmc.harvard.edu 